\title{
Am Fenster Klatschen als gescheiterter Versuch
}

Erschienen in: Sozialfiguren der Corona-Pandemie Von: Ulf Ortmann

\begin{abstract}
„Alle im Parkett klatschten und schrien ,Bravo!'. Dann stellte sich ein Mann in die Ecke. Im Orchester spielten die Zimbeln und Trompeten immer lauter, und dieser Mann begann mit nackten Beinen sehr hoch zu springen und dann wieder kleine Schritte zu machen. (Dieser Mann war Duport, der für diese Kunst sechzigtausend im Jahr erhielt.) Alle im Parkett, in den Logen und auf dem Olymp begannen zu klatschen und aus aller Kraft zu schreien, und der Mann blieb stehen und verbeugte sich lächelnd nach allen Seiten." 1
\end{abstract}

Natürlich hat sich diese Szene nicht im Verlauf der Corona-Pandemie ereignet. Tolstoi beschreibt hier den Applaus in einem Moskauer Theater kurz vor Napoleons Russlandfeldzug von 1812. Gleich nach Beginn der Pandemie, im Frühjahr 2020, klatschten Bürger*innen vielmehr an Fenstern, auf Balkonen und auf Terrassen für Beschäftigte in Krankenhäusern und Supermärkten - jedoch nicht nach dem Ende einer Aufführung „systemrelevanter“ Leistungen, sondern zu einem vereinbarten Zeitpunkt in den Abendstunden. Möglicherweise gibt es Nachbarschaften, in denen über den Beginn der Pandemie hinaus um 18 oder 21 Uhr geklatscht wurde und noch geklatscht wird. Als gesellschaftliches Phänomen ist das Klatschen allerdings aus den Hauptnachrichten verschwunden.

Warum kam die Figur der Klatschenden zu Beginn der Pandemie in den Fokus der Öffentlichkeit? Warum verlor sie diese Aufmerksamkeit nach wenigen Wochen? Und warum wird der Applaus heute gar - „Klatschen reicht nicht.“ - als heuchlerische Praktik angegriffen? ${ }^{2}$ Das Klatschen war ein ,figurativer 'Versuch, Beschäftigten Anerkennung entgegenzubringen, und ist - das ist meine These - in drei Hinsichten gescheitert. Erstens ist hier der Versuch gescheitert, Applaus als eine Praktik unter körperlich Anwesenden technisch zu vermitteln. Zweitens ist der Versuch gescheitert, die geleistete Arbeit als Handeln und Erleben in einer Ausnahmesituation zu verstehen, weil sich die Pandemie als gesellschaftliches Dauerproblem zeigt. Und schließlich ist der Versuch gescheitert, eine Beziehung zwischen Beschäftigten und einem Publikum zu etablieren, das weder als Patient*innen noch als Kund*innen zu erkennen ist: Als ob die Beschäftigten in Einzelhandel und Gesundheitswesen von einem anonymen Publikum Applaus für ihre Arbeit erwarten würden. ${ }^{3}$ 
Im Theater oder in der Konzerthalle richtet sich die Aufmerksamkeit des Publikums auf diejenigen, die auf der Bühne stehen. Im Fußballstadion sind die Blicke der meisten Zuschauer*innen auf den Rasen gerichtet. Die Aufmerksamkeit des Publikums, das an Fenstern und auf Balkonen klatschte, war dagegen nicht durch materielle Anordnungen (aus Beton, Stühlen oder Bühnen) auf bestimmte Akteur*innen gerichtet, sondern vielmehr durch einen parasoziologischen Diskurs sowie durch soziale Medien und öffentliche Berichterstattung vermittelt. In dieser Konstellation entstand Aufmerksamkeit - und verschwand genauso schnell, wie sie gekommen war.
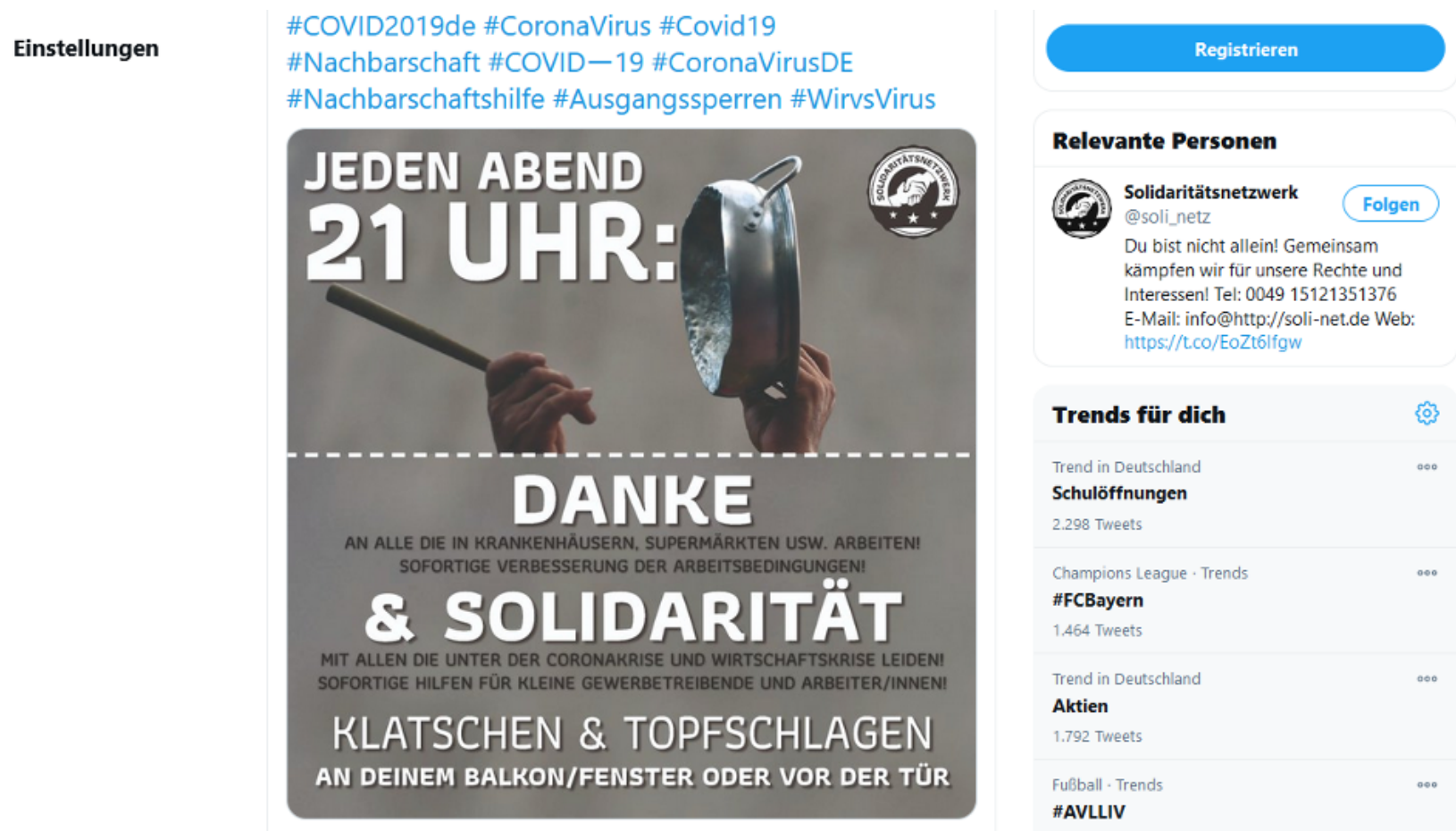

Abbildung 1: Aufruf zum Klatschen und Topf Schlagen auf Twitter. Screenshot von https://twitter.com/soli_netz/status/1240345532138172424

Mit Beginn der Pandemie entstand in Politik und Öffentlichkeit eine parasoziologische Systemtheorie, die im Kern besagte, dass Gesellschaft als ein Versorgungssystem mit verzichtbaren und unverzichtbaren Elementen begriffen werden kann. ${ }^{4}$ Unverzichtbare Elemente wurden als „systemrelevant“ bezeichnet; und systemrelevante Beschäftigte gelangten zu Beginn der Pandemie in den Fokus der Öffentlichkeit, weil sie zwei unterschiedliche Funktionen erfüllten. Sie stellten entweder die Grundversorgung „der Bevölkerung“ mit Gütern und Dienstleistungen sicher, zu denen auch Lebensmittel im engen Sinne zählten. Oder sie versorgten Covid-19 Erkrankte. Diese AdhocSystemtheorie wurde allerdings nur für kurze Zeit als Überzeugung gemeinsam geteilt. Denn wie jede Theorie - ich würde aber meinen: stärker als andere - reduzierte sie die Wirklichkeit. Nach wenigen Wochen im Lockdown war klar, dass Güter und Dienstleistungen kaum in verzichtbare und unverzichtbare Elemente zu zerlegen sind; und dass gerade die Gesundheitsversorgung nicht dauerhaft auf den gesellschaftlichen Umgang mit Covid-19 reduziert werden kann. Mit der skizzierten Systemtheorie verschwand eine wesentliche Begründung, auf systemrelevante Berufe die Aufmerksamkeit zu richten, weitestgehend aus dem öffentlichen Diskurs. 
Dass systemrelevante Arbeit in den ersten Wochen der Pandemie im Fokus der Öffentlichkeit war, war zum anderen bedingt durch die soziotechnische Konstellation, in der öffentliche Aufmerksamkeit entsteht und verschwindet. Selbstverständlich wurde und wird - systemrelevante Arbeit nicht in einer Situation bewältigt, in der Beschäftigte, Patient*innen bzw. Kund*innen und Publikum körperlich kopräsent sind. Das Publikum applaudierte Beschäftigten, die nicht vor ihren Fenstern und Balkonen standen; und die Klatschenden versammelten sich nicht, wie es applaudierendes Publikum sonst tut, sondern blieben zu Hause. Es bestand allenfalls Blickkontakt sowie eine akustische Verbindung zu den nächstgelegenen Balkonen, die lokal ein klatschendes Kollektiv (etwa eines Häuserblocks) entstehen lassen konnten - aber eben nicht in Anwesenheit der Beklatschten. Vielmehr war der Applaus technisch vermittelt: Die Arbeit auf den Intensivstationen und in den Supermärkten wurde in der Tagesschau gezeigt; über Instagram und Twitter riefen Teile des Publikums zum Applaus auf; und in sozialen Medien, Nachrichten und Zeitungen wurden auch die Klatschenden gezeigt. Egal, wie die Beschäftigten im Einzelhandel und im Gesundheitswesen diesen Applaus bewerteten: Der Applaus erreichte die Beschäftigten nicht, weil sie vor den Klatschenden auf der Bühne, auf dem Fußballplatz oder am Pult standen, sondern weil der Applaus Aufmerksamkeit in dieser soziotechnischen Konstellation erzeugte. Als das Klatschen allerdings seinen Neuigkeitswert verlor, wurde es - sofern überhaupt noch geklatscht wurde - nicht mehr in hohem Maße durch soziale Medien verbreitet, nicht mehr in der Tagesschau gezeigt und erreichte die systemrelevanten Akteur*innen kaum noch.

Stellen wir uns vor, dass über das Klatschen am Fenster wie über die Verbreitung des Coronavirus täglich berichtet worden wäre. Der Neuigkeitswert des Klatschens wäre dann nicht im Phänomen selbst sondern in seiner zahlenmäßigen Entwicklung begründet gewesen: „Binnen 24 Stunden wurden dem Robert-Koch-Institut 1,8 Millionen Klatschende gemeldet. Das sind 50.000 weniger als zum gleichen Zeitpunkt in der vergangenen Woche." Aber das ist absurd.

\section{Applaus für außerordentliche Leistungen, die doch dauerhaft erbracht werden}

Ein Publikum spendet Applaus unmittelbar nach einer Handlung von Sportler*innen, Schauspieler*innen, Sänger*innen oder Redner*innen. Je nach Umgebung ist Applaus auch spontan und unkoordiniert möglich (im Fußballstadion, bei den meisten Konzerten oder bei politischen Reden); in anderen Settings ist Applaus in der Regel nur nach dem Ende eines Auftritts oder in Pausen möglich (etwa beim Tennis, bei klassischen Konzerten oder bei wissenschaftlichen Vorträgen).

Das Klatschen nach dem Ende der Pandemie ist bis jetzt nicht möglich, weil die Pandemie noch nicht zu Ende ist. Aber auch spontaner Applaus oder Applaus während einer Unterbrechung ist kaum sinnvoll, wenn das Ende einer Aufführung nicht absehbar ist. Applaus setzt eine allen Beteiligten wenigstens ungefähr bekannte zeitliche Struktur der Situation voraus, in der außerordentliche Leistungen gezeigt werden: Ein Fußballspiel hat zwei Halbzeiten à 45 Minuten - und in dieser Zeit darf geklatscht werden. Ein Tennisspiel hat zwei bis fünf Sätze - und nach jedem Ballwechsel darf geklatscht werden. Und selbst die längste Oper bietet dem Publikum Gelegenheiten 
zum Applaus und nach Ende der Aufführung die Gelegenheit, den Saal zu verlassen. Dass es sich bei der Pandemie um eine zeitlich eng begrenzte Situation handelt, mag eine Erwartung gewesen sein, die maßgeblich zur Entstehung der Sozialfigur der Klatschenden beigetragen hat. Diese Erwartung hat sich allerdings nicht erfüllt.

Hinzu kommt: Offensichtlich scheint die Pandemie nicht den Zeithorizont zu bilden, vor dem die Beschäftigten Anerkennung erwarten. Die mannigfaltige Arbeit im Gesundheitswesen und im Einzelhandel ist außerordentlich, kunstfertig, sorgfältig, rechtzeitig und teilweise hochriskant. Applaus ist aber nicht die passende Form der Anerkennung - zumindest nicht aus der Perspektive derjenigen, die unter den Bedingungen der Pandemie systemrelevante Arbeit leisten und auch nach Ende der Pandemie leisten werden. Völlig zurecht fordern sie für ihre Leistung eine Anerkennung, die nicht wie Applaus an eine bestimmte Situation geknüpft ist, sondern die ihnen ebenso dauerhaft entgegengebracht wird, wie sie dauerhaft ihre Arbeit leisten. Dass das Klatschen am Fenster von den Beschäftigten nicht als warmer Applaus entgegengenommen wurde, hängt auch mit dem Zeitpunkt des Klatschens zusammen: Als ob in Krankenhäusern und im Einzelhandel nur dann außerordentliche Arbeit geleistet wird, wenn eine Pandemie ausbricht.

\section{Applaus als universelle Form der Anerkennung - wohl kaum}

Nachdem die Klatschenden in sozialen Medien, in den Zeitungen und im Fernsehen gezeigt wurden, verbeugten sich die Beschäftigten nicht lächelnd nach allen Seiten, wie es Duport gegenüber seinem Publikum in Tolstois „Krieg und Frieden“ macht. Im Gegenteil, stellvertretend für die Beschäftigten grenzten sich der DGB genauso wie der Bundesarbeitsminister vom gut gemeinten Applaus ab: „Klatschen reicht nicht." ${ }^{\text {Auch }}$ Pflegekräfte selbst wiesen im Verlauf der Pandemie öffentlich und mit Nachdruck auf den Applaus und dauerhafte Personalengpässe hin: „Euren Applaus könnt lhr Euch sonst wohin stecken."6

Dass der Applaus weniger als Belohnung angenommen wurde als möglicherweise erwartet, ist auch darauf zurückzuführen, dass die Beschäftigten in systemrelevanten Berufen keinen Applaus für ihre Arbeit erwarten. Zum einen arbeiten Ärzt*innen und Pflegekräfte, Kassierer*innen und Filialleiter*innen weder auf Bühnen noch am Podium. Ihre Arbeit ist nur teilweise und nur für Kund*innen oder Patient*innen sichtbar. Öffentliche Sichtbarkeit wurde aber durch das Klatschen am Fenster figuriert - und von den Beschäftigten im Fokus dankend abgelehnt. Zum anderen ist Applaus, auch wenn Arbeit mit technischen Mitteln sichtbar gemacht wird, nur dann eine passende Geste der Anerkennung, wenn die öffentlich gezeigte Leistung auch vor körperlich kopräsentem Publikum gezeigt werden kann. Wenn ich einen Vortrag über Zoom verfolge und zum Schluss die gelbe, klatschende Hand zeige, kann ich erwarten, dass die vortragende Person den Applaus wie von körperlich anwesendem Publikum entgegennimmt.

Arbeit, die nur bedingt sichtbar ist und für die kein Applaus erwartet wird, erfordert hingegen eine Anerkennung, die ohne öffentliche Aufmerksamkeit auskommt und von Kund*innen, Patient*innen, Kolleg*innen, Vorgesetzten oder Arbeitgeber*innen entgegengebracht wird. Angenommen, die Arbeitsbedingungen im Einzelhandel und im 
Gesundheitswesen hätten sich seit dem Frühjahr 2020 verbessert: Dann könnten wir darüber diskutieren, ob das Klatschen am Fenster zu diesen Verbesserungen beigetragen hat. Dass sich Arbeitsbedingungen in Gesundheitswesen und Einzelhandel seit Beginn der Pandemie verbessert haben, wird allerdings kaum jemand behaupten.

\section{Applaus als gescheiterte Figuration, um Anerkennung entgegenzubringen}

Applaus ermöglicht uns, denjenigen Anerkennung entgegenzubringen, die vor Publikum außerordentliche Leistungen erbringen. Und umgekehrt: Applaus ermöglicht uns, Anerkennung entgegenzunehmen, wenn wir vor Publikum reden, tanzen oder musizieren. Man denke etwa an Konzerte oder Theateraufführungen, die Musiker*innen oder Schauspieler*innen zu Beginn der Pandemie vor Balkonen und Fenstern gegeben haben. In diesen Situationen wurde Applaus vom Publikum gespendet und von den im Fokus der Aufmerksamkeit stehenden Akteur*innen entgegengenommen.

Beim Klatschen für systemrelevant Beschäftigte ist diese Übergabe von Anerkennung gescheitert: Daran, dass Applaus sich nicht ohne Weiteres technisch vermitteln lässt; daran, dass Applaus in einer zeitlich eng begrenzten Situation gespendet wird, aber die Pandemie vorerst kein Ende nimmt; und daran, dass die systemrelevant Beschäftigten weder ihre Arbeit vor Publikum leisten noch Applaus für ihre Arbeit erwarten.

Im Rückblick erscheint der Ausbruch der Pandemie als ein denkbar schlechter Zeitpunkt, um Applaus zu spenden. Denn Situationen, in denen vor Publikum in körperlicher Kopräsenz Geld verdient wird - im Theater, in der Konzerthalle oder im Fußballstadion - wurden im Verlauf der Pandemie zunächst stark eingeschränkt und dann Zug um Zug technisiert. Aber auch, wenn das Fensterklatschen als Versuch, Beschäftigten für geleistete Arbeit Anerkennung entgegenzubringen, gescheitert ist, könnte der Applaus einen Nebeneffekt haben, der den Beschäftigten in Einzelhandel und Gesundheitswesen irgendwann doch zugutekommen mag. Dass es um die Anerkennung von Arbeit in systemrelevanten Berufen nicht gut bestellt ist: Darauf haben das Klatschen am Fenster und die öffentliche Kritik daran - einmal mehr - aufmerksam gemacht.

\section{References}

1. Tolstoi, Lew: Krieg und Frieden (2018 [1868]): Krieg und Frieden, Erster Band, München: dtv, Seite 988.

2. https://www.dgb.de/presse/++co++0340debc-740c-11ea-b2bd-52540088cada, zuletzt geprüft am 5. Januar 2021.

3. Ich danke Julia Engelschalt, Julika Griem, Sebastian Hartwig, Sebastian Moser und Tobias Schlechtriemen für wertvolle Hinweise bei der Überarbeitung dieses Beitrags.

4. https://www.bmas.de/DE/Schwerpunkte/Informationen-Corona/Kurzarbeit/listesystemrelevante-bereiche.html, zuletzt geprüft am 5. Januar 2021.

5. https://www.tagesschau.de/ausland/corona-pflegekraefte-107.html, zuletzt geprüft am 5. Januar 2021. 
6. https://www.spiegel.de/gesundheit/coronakrise-krankenpflegerin-nina-boehmerfuehlt-sich-verheizt-a-48868e06-4aa7-4aef-873b-e43ca9d61110, zuletzt geprüft am 5. Januar 2021.

SUGGESTED CITATION: Ortmann, Ulf: Am Fenster. Klatschen als gescheiterter Versuch, in: KWI-BLOG, [https://blog.kulturwissenschaften.de/am-fenster/], 18.01.2021

DOI: https://doi.org/10.37189/kwi-blog/20210118-0830 


\section{DuEPublico}

Duisburg-Essen Publications online

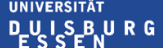

offen im Denken

ub $\mid \begin{aligned} & \text { universitäts } \\ & \text { bibliothek }\end{aligned}$

Dieser Text wird über DuEPublico, dem Dokumenten- und Publikationsserver der Universität Duisburg-Essen, zur Verfügung gestellt. Die hier veröffentlichte Version der EPublikation kann von einer eventuell ebenfalls veröffentlichten Verlagsversion abweichen.

DOI: $\quad$ 10.37189/kwi-blog/20210118-0830

URN: urn:nbn:de:hbz:464-20210118-095239-2

Alle Rechte vorbehalten. 